SU-ITP-99-20

hep-ph/9904432

September 6, 2018

\title{
Moduli Forces in String Models with Large Internal Dimensions
}

\author{
EDI HALYO ${ }^{*}$ \\ Department of Physics \\ Stanford University \\ Stanford, CA 94305
}

\begin{abstract}
We estimate the strength and range of forces mediated by string moduli in type I string models with two (or more) large internal dimensions. We find that forces mediated by twisted moduli which live on the brane world-volume can mediate forces which are orders of magnitude stronger than gravity with a range up to a milimeter. If they exist, these forces can be easily observed in present experiments. On the other hand, forces mediated by the dilaton and untwisted moduli are about a hundred times stronger than gravity and may be observed depending on their range.
\end{abstract}

\footnotetext{
* e-mail address: halyo@dormouse.stanford.edu
} 


\section{Introduction}

Recently there has been great interest in string theories at the $\mathrm{TeV}$ scale with large internal dimensions[1] ${ }^{\star}$. In this scenario, all Standard Model (or Minimally Supersymmetric Standard Model) degrees of freedom are confined to the $3+1$ dimensional noncompact brane world-volume whereas gravity propagates only in the compact bulk. The weakness of gravity with respect to the other interactions is a result of the large size of some of the internal dimensions. After compactification the four dimensional Newton constant is given by

$$
G_{N}=\frac{G_{d+4}}{R^{d}}
$$

where $R$ is the large compactification radius and $G_{d+4}=M_{d+4}^{-d-2} \sim(T e V)^{-d-2}$. Here $d$ denotes the number of large dimensions with all other compact dimensions of the string scale. (In string theory $d \leq 6$ necessarily.) The most interesting case which may also be the easiest to experimentally confirm or rule out is the $d=2$ case. Therefore, throughout this paper we will consider the case of two large dimensions unless it is explicitly stated. Surprisingly, this scenario cannot be easily ruled out either by accelarator experiments or by astrophysical observations[3]. The strongest bound on the higher dimensional Planck scale scale $M_{6}$ comes from the supernova 1987A giving $M_{6}>50 \mathrm{TeV}\left(M_{s}>4 \mathrm{TeV}\right)$ for two (three or more) large dimensions $[4]^{\dagger}$. In most of this paper we will assume that $M_{s} \sim M_{6}$ but this is not necessarily the case e.g. for small string coupling. During the last year various aspects of this scenario have been explored including phenomenology[6], collider signatures[7] and cosmology[8].

An immediate consequence of this scenario is the change in the gravitational force law at a length scale which corresponds to the size of the large dimensions $[9,10,11]$, e.g for two large dimensions with $M_{6} \sim M_{s} \sim 50 \mathrm{TeV}$ this corresponds to $R \sim 0.4 \mu m$. At this scale, we expect the gravitational force law to

* For earlier work along this line see [2].

$\dagger$ However see [5] for a model dependent higher bound of $M_{6}>110 \mathrm{TeV}$. 
change from $1 / r^{2}$ to $1 / r^{4}$. However, this length scale is too small for present and planned experiments to probe[12]. It would be desirable if this scenario had an observable signature at larger length scales such as tens of microns which can be probed by these experiments. In this paper, we consider forces mediated by moduli in string models with large internal dimensions. In these models, SUSY must effectively be broken at or below $M_{s} \sim T e V$ and as a result the Compton wavelength of these moduli are rather large, e.g. in the micron range. This is also the range of the new moduli force which can be seen as a correction to gravity.

String models with large internal dimensions can be concretely realized in type I orbifold compactifications with D-branes or equivalently by type IIB orientifold compactifications[13]. In type I string models, a priori there are three kinds of moduli which can mediate new forces, the dilaton $S$, untwisted moduli $T_{i}$ and twisted moduli $M_{i}$. We argue that $S$ and $T$ which are bulk fields mediate forces with a range of microns if SUSY is broken on the brane (by some field theoretic nonperturbative phenomenon like gaugino condensation). However, their interactions with matter cannot be much stronger than gravity and therefore not easily observable by the present and planned experiments. On the other hand, twisted moduli $M$ which live on the (orbifold) fixed planes can mediate forces many orders of magnitude stronger than gravity. If the branes coincide with the fixed planes, these moduli are confined to the brane world-volume and they have interactions with matter which are suppressed by $M_{s}$ rather than $M_{P}$. The integration over the large volume factor which is the source of the $M_{P}$ suppression of graviton, $S$ and $T$ interactions with matter is missing in this case. Thus, with respect to gravity their interactions with matter are enhanced by the very large factor $\left(M_{P} / M_{s}\right)^{2}$. Unfortunately, due to the same enhancement the twisted moduli also get very large i.e. $\sim M_{s}$ masses. As a result, their interactions with matter are completely negligible in the micron range. On the other hand, these heavy twisted moduli can give rise to interesting phenomenology[15].

\footnotetext{
$\ddagger$ The same fields give rise to acceptable D-term inflation in type I string models[14].
} 
Fortunately, there are other possible configurations. For example, there may be a fixed compact torus under the orbifold group. Then the twisted moduli also live on this torus in addition to the brane world-volume. In this case, the coupling of the twisted moduli to matter is suppressed by the square root of the volume of the torus in string units. If the fixed torus is large enough the moduli masses can be small enough to mediate a force with a macroscopic range. In addition, the moduli the coupling to matter can be suppressed (relative to the enhancement $\left.\left(M_{P} / M_{s}\right)^{2}\right)$ to give forces much stronger than gravity which are not experimentally ruled out. In this paper, we estimate the twisted moduli masses and the strength of the force mediated by them in generic type I string models. We show that for realistic values of the parameters such as compactification radii, supersymmetry (SUSY) breaking scale and $M_{s}$ the force mediated by $M$ can be long range and orders of magnitude stronger than gravity.

This paper is organized as follows. In section 2 we derive the general formulas for forces mediated by string moduli and mention how these apply to type I string models. Section 3 is a brief review of type I string models which realize the scenario with large internal dimensions. In section 4 we estimate the magnitude of the modulus force and the moduli masses in a number of different scenarios. We show that forces mediated by twisted moduli are generically much stronger than gravity and other moduli forces. Section 5 is a discussion of our results.

\section{Moduli Forces in String Theory}

In heterotic string models with TeV scale SUSY breaking the masses of string moduli such as the dilaton $S$ and untwisted moduli $T_{i}$ is given by $m_{S, T} \sim$ $\mathrm{TeV}^{2} / M_{P} \sim 10^{-12} \mathrm{GeV}[9]$. This corresponds to a Compton wavelength of milimeters. Thus, in these models string moduli can mediate forces with a macroscopic range. Such a new modulus force modifies the gravitational interaction as[10]

$$
V(r)=-G_{N} \frac{m_{1} m_{2}}{r}\left(1+G_{S, T}^{2} e^{-r / \lambda_{S, T}}\right)
$$

where $G_{S, T}^{2}$ gives the relative strength of the modulus force to gravity and $\lambda_{S, T}$ is 
the range of the modulus force fixed by the Compton wavelength of the modulus.

The couplings of $S$ and $T$ to matter arise from the dependence of the QCD coupling constant on the dilaton (at tree level) and the moduli (at one loop)

$$
\frac{1}{g_{3}^{2}}=k_{3} \operatorname{Re} S+f(T)
$$

where $k_{3}=1$ and $f(T)$ gives the one-loop corrections which depend on the moduli. On dimensional grounds the nucleon mass can be written as

$$
m_{N} \sim \exp \left(-8 \pi^{2} / b g_{3}^{2}\right) M_{P}
$$

Here $b$ is the first coefficient of the QCD $\beta$-function. The dilaton-nucleon coupling is given by $[10,9]$

$$
G_{S}=\frac{\partial m_{N}}{\partial S} \frac{\partial S}{\partial S_{c}}
$$

with a similar expression for the modulus-matter coupling. The second term on the right hand side takes into account the fact that moduli generically have nontrivial Kahler potentials and the force is mediated by the canonically normalized modulus field $S_{c}$. Due to the large hierarchy between $M_{P}$ and $m_{N}$ the dilaton coupling to matter is rather large compared to gravity; e.g. $G_{S}^{2} \sim 10^{3}$. The modulus coupling is much weaker than gravity, e.g. $G_{T}<<1$ because it arises at one loop and is suppressed by a factor of $1 / 16 \pi^{2}$. (However in models with Scherk-Schwarz SUSY breaking at the $\mathrm{TeV}$ scale the modulus coupling can be as strong as gravity[9].)

In string models with large internal dimensions the situation is slightly different. First, the string scale (or rather $M_{d+4}$ ) is not large but around the TeV scale. Taking into account the bounds from supernova 1987A we assume $M_{6} \sim M_{s}>50 \mathrm{TeV}$. If SUSY is broken only on the brane this gives a Compton wavelength of $\lambda_{S, T}<0.4 \mu \mathrm{m}$ for two large dimensions. Thus, the range of the modulus force is much shorter than a milimeter. Second, not every modulus necessarily couples to observable matter. In the heterotic string models mentioned above this 
was the case due to the fact that both $S$ and $T$ appeared in the expression for $g_{3}$. We will see that in type I string models either $S$ or $T$ but not both couple to matter. Moreover, in these models the couplings of $S$ and $T$ to matter are more than an order of magnitude weaker than the corresponding forces in heterotic models since there are only four to five orders of magnitude between $m_{N}$ and $M_{s}$. We conclude that in this scenario forces mediated by untwisted moduli such as $S$ and $T$ are short range and not strong enough and therefore probably cannot be observed in present or planned experiments. (Note however that for small string coupling or for more than two large dimensions a lower string scale is possible. Then, the range of the force would be longer and it may be observable.)

Finally, there can be twisted moduli which cannot propagate in the bulk as $S$ and $T$ but live on the (orbifold) fixed planes. If the branes are at the fixed point interactions of twisted moduli with matter are suppressed only by $M_{s}$ rather than $M_{P}$. As a result, their couplings to matter are many orders of magnitude stronger than gravity. Unfortunately, in this case moduli masses are unsuppressed and $\sim M_{s}$. Therefore these forces are completely negligible at the micron range. However, if there is a fixed torus of the orbifold group the moduli coupling to matter and their masses are suppressed by the square root of the volume of the torus. For a large enough torus this may lead to strong moduli interactions with a long range. In these cases the twisted moduli may have long range interactions which are orders of magnitude stronger than gravity. In type I string models there are twisted moduli $M$ with the above properties. Their VEVs are related to the blowup of the orbifold singularities which smoothes out the singular compactification geometry. In section 4 we will estimate the force mediated by these twisted moduli and their masses for a number of different scenarios.

\section{Type I String Theory or Orientifolds of Type IIB Strings}

Four dimensional type I string compactifications with $N=1$ supersymmetry can be obtained by orientifolds of type IIBstrings[13]. We start with a type IIB 
string theory in $D=10$ and mode it out by the world-sheet parity transformation $\Omega$. This gives a type I string theory in $D=10$ with gauge group $S O(32)$. The gauge group arises from the 32 D9 branes required for tadpole cancellation. This type I string theory is further compactified on an orbifold of $T^{6}=T^{2} \times T^{2} \times T^{2}$ (with radii $R_{1,2,3}$ ) i.e. on $T^{6} / \Gamma$ where $\Gamma$ is a discrete group such as $Z_{n}$ or $Z_{n} \times Z_{m}$ resulting in a $D=4$ theory with $N=1$ supersymmetry and chiral matter content. The above construction has only D9 branes but by considering more elaborate orientifolds one can obtain models with two types of branes in the theory. For example, if $O_{i}$ denotes reflection of $T_{i}^{2}$ then one can mode $T^{6}$ by $(-1)^{F_{L}} \Omega O_{i}, \Omega O_{i} O_{j}$ or $(-1)^{F_{L}} \Omega O_{i} O_{j} O_{k}$. Here $F_{L}$ is the left handed world-sheet fermion number. The above modings result in D7, D5 and D3 branes respectively. Due to the requirement for $N=1$ SUSY only two sets of branes can appear simultaneously; either D9 and D5 branes or D3 and D7 branes[13]. We denote D5 branes as $5_{i}$ if the branes wrap around $T_{i}^{2}$ and the D7 branes as $7_{i}$ if they wrap around $T_{j}^{2} \times T_{k}^{2}$. The number of each kind of brane is fixed again by tadpole cancellation. On the two different kinds of branes $\left(D p\right.$ and $\left.D p^{\prime}\right)$ there are gauge multiplets from strings with both ends on the same kind of brane (i.e. $p p$ or $p^{\prime} p^{\prime}$ strings), giving two gauge groups, $G_{p}$ and $G_{p^{\prime}}$. Different polarizations of the same open strings also give matter multiplets in the adjoint representation of the gauge group. In additon, there are matter fields which arise from strings with ends on different kinds of branes (i.e. $p p^{\prime}$ strings) in the bifundamental representation. Newton's constant is given by

$$
G_{N}=\frac{1}{M_{P}^{2}}=\frac{g_{I}^{2}}{8 M_{I}^{8} R_{1}^{2} R_{2}^{2} R_{3}^{2}}
$$

Here $g_{I}$ is the type I string coupling constant and $M_{I}=\alpha_{s t r}^{-1 / 2}$ is the type I string scale.

We now consider the two possibilities, i.e. models with D3-D7 branes and D9-D5 branes separately[13].

a) Models with D3 and D7 branes: These are obtained by moding out type IIB string theory by the group generated by $(-1)^{F_{L}} \Omega O_{i}$ and $(-1)^{F_{L}} \Omega O_{i} O_{j} O_{k}$. There 
are two gauge groups $G_{3}$ and $G_{7}$ living on the D3 and D7 branes. The matter content of these models arises from strings stretched between different branes, i.e. 33, 37, 73 and 77 strings (Here and below we omit the indices $i, j$ for notational simplicity.). We denote these fields by $M^{33}, M^{37}, M^{73}, M^{77} . M^{33}$ and $M^{77}$ are in the adjoint representation of the respective gauge groups, $G_{3}$ and $G_{7}$, whereas $M^{37}$ and $M^{73}$ are in the bifundamental representation. In realistic models there are Wilson lines which break the gauge group and project out part of the matter. The tree level superpotential generically contains the terms[16]

$$
W=g_{3}\left(M^{33} M^{73} M^{37}\right)+g_{7}\left(M^{77} M^{37} M^{73}\right)
$$

The gauge couplings are given by $\left(\alpha_{i}=g_{i}^{2} / 4 \pi\right)$

$$
\alpha_{3}=\frac{g_{I}}{2} \quad \alpha_{7_{i}}=\frac{g_{I}}{2 M_{I}^{4} R_{j}^{2} R_{k}^{2}}
$$

There are three kinds of moduli; the dilaton $S$ and the untwisted moduli $T_{i}$ are defined as

$$
S=\frac{2}{g_{I}}+i \theta
$$

and

$$
T_{i}=\frac{2 R_{j}^{2} R_{k}^{2} M_{I}^{4}}{g_{I}}+i \eta_{i}
$$

where $\theta$ and $\eta_{i}$ are untwisted Ramond-Ramond fields. The Kahler potential for $S$ and $T_{i}$ is given by

$$
K\left(S, \bar{S}, T_{i}, \bar{T}_{i}\right)=-\log (S+\bar{S})-\Sigma_{i} \log \left(T_{i}+\bar{T}_{i}\right)
$$

The third kind of moduli are the twisted moduli denoted by $M_{i}=\phi_{i}+i \psi_{i}[13] . \phi_{i}$ and $\psi_{i}$ are twisted NS-NS and R-R fields respectively. The VEV of $\phi_{i}$ parametrize the blowup of the orbifold singularities or the smooothing of the compact space. 
The Kahler potential for $M_{i}$ is not known but we will assume that it is canonical. In the presence of $M$ the connection between the gauge couplings and the moduli are given by[13]

$$
\frac{1}{\alpha_{3}}=R e S+s_{3} M
$$

and

$$
\frac{1}{\alpha_{7_{i}}}=R e T_{i}+s_{7_{i}} M
$$

Here the $s_{3,7_{i}}$ are constants of $O(1-10)$. In these models, there is fixed torus $T_{j}^{2} \times T_{k}^{2}$ of the of the orbifold group $O_{i}$. Therefore, twisted moduli corresponding to this twist live in eight dimensions along the D7 branes. The orbifold group $O_{i} O_{j} O_{k}$ has only fixed points and therefore twisted moduli corresponding to these live in four dimensions parallel to the D3 branes.

b) Models with D5 and D9 branes: These are obtained by moding out type IIB string theory by the group generated by $\Omega$ and $\Omega O_{i} O_{j}$. The generic gauge and matter content and the superpotential are very similar to the previous case with the substitution $3 \rightarrow 9$ and $5_{i} \rightarrow 7_{i}$. The reason for this is the fact that the two kinds of models are related to each other by $\mathrm{T}$ duality along all the compact dimensions. In this case the gauge couplings are

$$
\alpha_{9}=\frac{g_{I}}{M_{I}^{6} R_{1}^{2} R_{2}^{2} R_{3}^{2}} \quad \alpha_{5_{i}}=\frac{g_{I}}{M_{I}^{2} R_{i}^{2}}
$$

Now, the dilaton and untwisted moduli are defined as

$$
S=\frac{2 M_{I}^{6} R_{1}^{2} R_{2}^{2} R_{3}^{2}}{g_{I}}+i \theta
$$

and

$$
T_{i}=\frac{2 M_{I}^{4} R_{j}^{2} R_{k}^{2}}{g_{I}}+i \eta_{i}
$$

The Kahler potential for $S$ and $T_{i}$ remains the same. The dependence of the $G_{9}$ and $G_{5}$ gauge couplings on the moduli is also given by eqs. (12) and (13) with the 
substitution $3 \rightarrow 9$ and $5_{i} \rightarrow 7_{i}$. Once again there are twisted moduli $M_{i}$ which are related to the blowup of the singular compact space. Twisted moduli which correspond to the twist $\Omega$ live in ten dimensions, i.e. they can move in the whole space. The ones arising from $O_{i} O_{j}$ live in six dimensions since there is only a fixed $T_{k}^{2}$ under this twist.

\section{Moduli Forces in String Models with Large Dimensions}

In this section we estimate the strength of the moduli forces in the scenario with two large dimensions within the framework of type I string theory. We assume that $M_{s} \sim M_{6} \sim 50 \mathrm{TeV}$ and the SUSY breaking scale is $\Lambda \sim 10 \mathrm{TeV}$. We take the SUSY breaking scale to be less than the string scale in order not to generate a new hierarchy. However it is easy to show that our results hold also for the case $\Lambda \sim M_{s}$. Using eq. (1) we find that in this case the two large compact dimensions are of the size $R \sim 4 \times 10^{8} \mathrm{GeV}^{-1} \sim 0.4 \mu \mathrm{m}$. So there are two $T^{2}$ s of size $M_{s}^{-2}$ and one of size $R^{2}$.

First consider the models with D3 and D7 branes. We assume that we live on the D3 branes therefore we need $g_{3} \leq 1$. Thus from eq. (8) we have $g_{I} \leq 1 / 10$ (or $S \sim 20$ ) i.e. we are in perturbative regime of type I string theory. We also assume that there is one other set of D7 branes wrapping the $T^{2} \times T^{2}$ of string scale. (Otherwise there are D7 branes wrapeed around the large dimensions and therefore light Kaluza-Klein states on the branes which are not desirable.) From eq. (10) we find that one of the three untwisted moduli is around $T \sim 2 / g_{I}$ whereas the other two are very large, $T_{i} \sim 4 \times 10^{26}$.

In models with D9 and D5 branes the situation is similar. Once again we have D9 branes and one set of D5 branes wrapping a $T^{2}$ of the string size. Now from eq. (15) we have $S \sim 4 \times 10^{26}$ giving a very small $g_{9}$. Thus, we assume that our world lives on the three noncompact dimensions of the D5 brane world--volume. In this case there two possibilities. If the two large dimensions belong to the same $T^{2}$ then there are two untwisted moduli with $T_{i} \sim 2 / g_{I}$ and one with $T \sim 4 \times 10^{26}$. 
On the other hand, if the two large dimensions belong to two different $T^{2} \mathrm{~s}$ then there is one modulus with $T \sim 2 / g_{I}$ and two large moduli of size $T_{i} \sim 2 \times 10^{13}$.

In these models there are two possibilities for SUSY breaking. SUSY can be broken either on the brane or in the bulk. (Of course it can also be broken both in the bulk and on the brane but this case is obtained from the two above.) If SUSY is broken on the brane by a mechanism like gaugino condensation at a scale $\Lambda$ then this breaking is mediated to the observable sector either by gauge interactions or by nonrenormalizble interactions suppressed by powers of $M_{s}$. Then $m_{S, T} \sim$ $\Lambda^{3} / M_{s} M_{P}$ since interactions of the bulk modes with the brane are suppressed by $M_{P}$. This is a small mass and therefore $S$ and $T$ have the potential to mediate macroscopic range forces. On the other hand, if SUSY is broken in the bulk by an F-term of $O\left(M_{s} M_{P}\right)$ then $S$ and $T$ get very large i.e. $\sim M_{S}$ masses. Therefore their interactions with matter are very short range and completely negligible at the micron range.

We can now estimate the strength of the forces mediated by the untwisted moduli when SUSY is broken on the brane only. The moduli masses are $m_{S, T} \sim$ $\Lambda^{3} / M_{s} M_{P} \sim 2 \times 10^{-11} \mathrm{GeV}$ giving a range of $\lambda_{S, T} \sim 50 \mu \mathrm{m}$. In models with D3 and D7 branes $T$ does not couple to observable matter since it does not appear in the expression for $g_{3}$. $S$ on the other hand does couple to matter. Due to the nontrivial Kahler potential for $S$ given by eq. (11) the canonical dilaton is given by $S_{c} \sim \log S$ (at least for the real part of $S$ which we are interested in). Using $M_{s} \sim 50 \mathrm{TeV}$ and eq. (4) for $m_{n}$ and eq. (5) we get $G_{s} \sim 10$. Thus the dilaton force in this case is about two orders of magnitude stronger than gravity with a range of $50 \mu \mathrm{m}$. In models with D9 and D 5 branes our world lives on the D 5 branes and therefore $S$ does not couple to matter. The only untwisted modulus $T$ that appears in $g_{3}$ is the one with a small VEV, i.e. $T \sim 2 / g_{I}$. The canonical modulus field is given by $T_{c} \sim \log T$ and the analysis is identical to that of $S$ above resulting in a force stronger than gravity mediated by the untwisted modulus. If the range of the modulus force is $\sim 50 \mu \mathrm{m}$ then it becomes of the order of gravity around $200 \mu \mathrm{m}$ which is around what present experiments can detect. We conclude that 
for this choice of parameters forces mediated by $S$ and $T$ are about two orders of magnitude stronger than gravity and may be observed. Of course, if we assume $\Lambda \sim M_{s}$ the range of the force becomes much smaller e.g. $\sim 0.4 \mu m$ and then it cannot be observed by the present experiments. (However, note that we assume $M_{s} \sim M_{6}$ here. If the string coupling is small or there are more than two large dimensions so that $M_{s} \sim 3-10 \mathrm{TeV}$ then this force with $G_{S, T}^{2} \sim 100$ has a range around $10-100 \mu m$ which can easily be detected.) Above the strength of the $S$ and $T$ moduli forces is normalized by the Newton constant since these fields live in the bulk. As a result, their couplings to matter which live on the brane are suppressed by the large volume factor, i.e. $M_{P}$.

We now turn to the twisted moduli $M$ which are present in these models. The coupling of $M$ to matter arises from the dependence of $g_{3}$ on $M$ given by eq. (12). Then the nucleon mass becomes

$$
m_{N} \sim \exp \left(-8 \pi^{2} / b g_{3}^{2}\right) M_{s} \sim \exp \left[-8 \pi^{2} \operatorname{Re}\left(S+s_{i} M\right) / b\right] M_{s}
$$

In some models $s_{i}=b \sim 10$, in any case these constants are of the same magnitude[17]. Therefore, for simplicity in our estimates we will take them to be equal. Note that the coefficient $s_{i}$ is may be a large number (in which case the VEV of $M$ has to be smaller than $M_{s}$ ). As we will see this is the origin of the strong coupling of $M$ to matter. If $s_{i} \sim 1$ coupling $M$ to matter would be as strong as those of $S$ or $T$ only. The twisted moduli $M$ live on the fixed planes and cannot propagate in the bulk. If the D-branes coincide with the fixed planes the twisted moduli live on the branes. As a result, their interactions with matter are suppressed by $M_{s}$ and not $M_{P}$ giving an enhancement of $\left(M_{P} / M_{s}\right)^{2} \sim 4 \times 10^{26}$ with respect to gravity. Using eq. (17) for $m_{N}$ we find that $G_{M} \sim(2 s / b) \times 10^{15}$ which gives a force thirty orders of magnitude stronger than gravity at its peak. The mass of $M$ is independent of where SUSY is broken. If SUSY is broken on the brane then $m_{M} \sim M_{s}$ and the moduli force has a very short range. On the other hand, if SUSY is broken in the bulk with $F \sim M_{s} M_{P}$ (in order to give large masses to superparticles) then again 
$m_{M} \sim M_{s}$. Therefore, when the branes overlap with the fixed planes, even though the modulus force is very strong at its peak, it is negligible at the micron range due to its extremely short range.

We found that the modulus mass is generically $\sim M_{s}$ leading to a very short range for the force mediated by $M$. Our arguments above are valid for the orbifolds with twists that have only fixed points but no fixed tori in the compact directions. If there are such fixed tori then the untwisted moduli can move on them in addition to the noncompact brane world--volume. For example, consider an orbifold twist with a fixed $T^{2}$ such as $(-1)^{F} \Omega O_{i} O_{j}$ we considered in the previous section for type I string models. Then the corresponding twisted modulus lives in six dimensions including the compact torus $T_{k}^{2}$ which is invariant under this twist. As a result, its four dimensional effective interactions with matter living on the brane are suppressed by the square root of the volume of the torus in string units. We find that the interaction with matter is given by

$$
G_{M} \sim\left(\frac{8 \pi^{2} g_{I}^{2} s}{b}\right)\left(\frac{M_{P}}{M_{s}}\right)\left(\frac{1}{R^{2} M_{s}^{2}}\right)^{1 / 2}
$$

and the modulus mass is

$$
m_{M} \sim g_{I}\left(\frac{\Lambda^{3}}{M_{s}^{2}}\right)\left(\frac{1}{R^{2} M_{s}^{2}}\right)^{1 / 2}
$$

Now, if the fixed torus has large dimensions there is a large suppression in the modulus mass which can give a long range for the modulus force. Since the force is thirty orders of magnitude stronger than gravity before this suppression we expect it to still be much stronger than gravity with the volume suppression. It is easy to see that if the fixed torus has two large dimensions

$$
G_{M} \sim\left(\frac{8 \pi^{2} g_{I}^{2} s}{b}\right)
$$

Thus in this case we find $G_{M} \sim 80$ giving a force $6 \times 10^{3}$ times stronger than gravity (for the realistic choice of parameters $s \sim b, g_{I} \sim 1$ ). We stress that the 
force mediated by $M$ is stronger than those mediated by $S$ or $T$ due to large value of $s_{i} \sim b \sim 10$. Also it is easy to see that the volume suppression of the interaction is exactly the factor that converts $1 / M_{s}^{2}$ to $1 / M_{P}^{2}$ so that the force is enhanced only by the factor in eq. (20).

The range of the force is given by the Compton wavelength of the modulus $\lambda_{M} \sim M_{s} M_{P} / \Lambda^{3}$ which is always larger than the size of the large dimension $R \sim M_{P} / M_{s}^{2}$ for $\Lambda<M_{s}$. In the above case $\lambda_{M} \sim 50 \mu m$. The force has range of $\sim 100 R$ which is about the range of the force mediated by $S$ or $T$. It becomes of gravitational strength around $450 \mu \mathrm{m}$ which is easily observable by the present experiments. This is an effect much more dominant than the change in the gravitational force or the force mediated by untwisted moduli.

Above we assumed that the SUSY breaking scale $\Lambda$ is smaller than $M_{s}$ in order not to generate a new hierarchy problem in light of the large string scale. However one can show that even if $\Lambda \sim M_{s}$ the modulus force is much stronger than gravity. The strength of the moduli force at its peak is given by $G_{M}^{2} \sim\left(8 \pi^{2} s g_{I}^{2} / b\right)^{2}$ and is independent of the SUSY breaking scale or the range of the moduli force. When $\Lambda \sim M_{s}$, however the range of the force $\lambda_{M}$ is equal to the size of the large dimension $R$. For many reasonable values of the parameters $G_{M}^{2}$ above is much larger than unity giving a force a few orders of magnitude stronger than gravity.

We also assumed that $M_{s} \sim M_{6}$ which is not the case for small string coupling. The force mediated by twisted string moduli can be much stronger than gravity even if $M_{s}<M_{6}$. For example, take $M_{s} \sim 10 \mathrm{TeV}$ and $\Lambda \sim 5 \mathrm{TeV}$ for $M_{6} \sim$ $50 \mathrm{TeV}$. The size of the large dimensions is $R \sim 10 \mu \mathrm{m}$ whereas the range of the modulus force is $\lambda_{M} \sim 80 \mu m$ for two large dimensions. The strength of the force at this distance is $G_{M}^{2} \sim 6 \times 10^{3}$. This force becomes of the gravitational strength at $\sim 0.8 \mathrm{~mm}$. Thus we find that even if $M_{s}<M_{6}$ there can be moduli forces which are much stronger than gravity which are easily observable.

Most of the above analysis was done for two large dimensions for which there is a model independent bound on the six dimensional Planck scale $M_{6}>50 \mathrm{TeV}$. For 
more than two large dimensions this bound relaxes significantly, i.e. for three large dimensions $M_{7}>4 \mathrm{TeV}$ and for four and more large dimensions $M_{d}>1 \mathrm{TeV}[4]$. It is easy to show that the strength of the force and its range are independent of the number of large dimensions. Assuming that the twisted moduli live in $4+d$ dimensions the range of the force (for $d$ large dimensions) is given by

$$
\lambda_{M} \sim\left(\frac{M_{s}^{2}}{\Lambda^{3}}\right)\left(\frac{1}{R^{d} M_{s}^{d}}\right)^{-1 / 2}
$$

where $R$ is given by eq. (1) to be $R^{d} \sim M_{P}^{2} / M_{s}^{d+2}$. We see that the range is $\lambda_{M} \sim M_{s} M_{P} / \Lambda^{3}$ independent of the number of large dimensions. In particular for $\Lambda \sim M_{s}$ we find $\lambda_{M} \sim M_{P} / M_{s}^{2}$. On the other hand, the strength of the moduli force in $d$ dimensions is given by

$$
G_{M} \sim\left(\frac{8 \pi^{2} g_{I}^{2} s}{b}\right)\left(\frac{M_{P}}{M_{s}}\right)\left(\frac{1}{R^{d} M_{s}^{d}}\right)^{1 / 2}
$$

We find that for all $d, G_{M} \sim\left(8 \pi^{2} g_{I}^{2} s / b\right)$ giving a force $\sim 6 \times 10^{3}$ times stronger than gravity. We see that independent of the number of large dimensions if $\Lambda \sim M_{s}$ then the range of the force $\lambda_{M} \sim R$. However, the the moduli force is about three orders of magnitude stronger than gravity at this scale and therefore a much larger effect.

For example, consider the case of three large dimensions with $M_{s} \sim M_{7} \sim \Lambda \sim$ $4 \mathrm{TeV}$ [4]. Then the large radii are of size $60 \mu \mathrm{m}$ and the range of the moduli force is also $\lambda_{M} \sim 60 \mu m$ (and even longer if we take $\Lambda<M_{s}$ ). Now $G_{M}^{2} \sim\left(8 \pi^{2}\right)^{2} \sim$ 6500 and it becomes of order unity at $\sim 0.6 \mathrm{~mm}$. Thus the effect of the moduli force is much larger than the change in gravity and easily observable in present experiments. With more than three large dimensions the bound on $M_{d} \sim M_{s}$ is relatively small and all effects occur around the milimeter scale. Since forces of gravitational strength are experimentally excluded above a milimeter it is difficult to have moduli forces stronger than gravity at this scale. However, it is clear that if for $d \geq 3$ the string scale can be pushed up by collider or astrophysical constraints it will be easy to have moduli forces stronger than gravity. 
Of course for more than two large dimensions, the twisted moduli do not have to live on all the large dimensions. For example there can be three large dimensions but the same orbifold twist considered above would still have only a fixed $T^{2}$. Then $M$ live in six dimensions as before; however now the size of the large dimension $R$ is different. In this case we have

$$
G_{M} \sim\left(\frac{8 \pi^{2} g_{I}^{2} s}{b}\right)\left(\frac{M_{P}}{M_{s}}\right)^{1 / 3}
$$

Thus we get $G_{M}^{2} \sim 10^{10}$. The range of the force is

$$
\lambda_{M} \sim\left(\frac{M_{s}^{2}}{\Lambda^{3}}\right)\left(\frac{M_{s}}{M_{P}}\right)^{2 / 3}
$$

This gives $\lambda_{M} \sim 0.1 \mu m$ which is rather small. We find that this possibility leads to a very strong force with a very short range which is negligible compared to the forces mediated by the untwisted moduli. However, the three large dimensions do not have to be isotropic. For example, if the area of the fixed $T^{2}$ is $R_{1} R_{2} \sim 10^{20} \mathrm{GeV}^{-2}$ (with the third large dimension of size $R_{3} \sim 80 \mathrm{GeV}$ ) we find that the range of the force is $\lambda_{M} \sim 10^{10} \mathrm{GeV}^{-1} \sim 10 \mu \mathrm{m}$ and the strength of the force is given by $G_{M}^{2} \sim 3 \times 10^{5}$. We see that the anisotropic large dimensions have the potential to give rise to very strong forces with a long range.

We implicitly considered untwisted moduli fields $M$ with canonical Kahler potentials. However, the Kahler potential for $M$ is probably not canonical but of the form

$$
K\left(M_{i}, \bar{M}_{i}\right)=\frac{M_{i} \bar{M}_{i}}{\left(T_{i}+\bar{T}_{i}\right)^{n_{i}}}
$$

Here $n_{i}$ are positive integers and $T_{i}$ are the untwisted moduli. In this case, we have observable forces only if the moduli $T_{i}$ in the Kahler potential have small i.e. $O(1)$ values that is they correspond to radii of string size. Above we saw that in all models there are such untwisted moduli. Otherwise, there is a very large field renormalization due to the above Kahler potential $M$ is renormalized by a 
factor of $\left(2 \times 10^{13}\right)^{n_{i}}$. This makes the already very strong modulus force many orders of magnitude stronger; however it also renormalizes the modulus mass by the same factor. As a result, the force becomes extremely short range and therefore completely negligible at the micron range.

\section{Conclusions}

In this paper we have shown that type I string models with large internal dimensions may have light moduli which can mediate forces orders of magnitude stronger than gravity with a macroscopic range. We found that the untwisted moduli $S$ and $T$ can mediate forces about 100 times gravity with a range of microns. On the other hand, the twisted moduli $M$ which live on the branes can mediate forces orders of magnitude stronger than gravity with a range that is longer than the size of the large dimensions. As a result, these effects are much easier to probe by the present and planned experiments than the change in the gravitational force. This is especially the case for two large dimensions with a string scale of $50 \mathrm{TeV}$ which puts the size of the large dimensions out of experimental reach.

The smallness of the moduli masses is the reason for the long range of the force. The mass is small due to the suppression of the moduli interactions with matter by the square root of the volume of a large torus on which the fixed moduli can move. The same suppression factor also reduces the huge enhancement in the moduli interactions with matter. In fact if the twisted moduli move on all the large dimensions this factor takes $1 / M_{s}$ to $1 / M_{P}$ giving gravitational strength interactions up to the enhancement coming from the moduli coupling to the nucleons. We saw that if the string parameter $s_{i} \sim b$ the force is orders of magnitude stronger than gravity. We find that there can be moduli forces with a range of tens to hundreds

of microns and strength of four orders of magnitude stronger than gravity for a wide range of parameters of the string model. If the orbifold group of the string model does not have fixed tori or these tori are string size then the range of the moduli force is extremely small and it cannot be detected. 
The above results are quite robust. They hold for realistic values of the string scale, SUSY breaking scale and other parameters of the string model. In particular, they are valid for any number of large internal dimensions as we showed above. The strength of the moduli interactions with matter is independent of the SUSY breaking scale and the number of dimensions (but depends on the bound on the string scale and on $s_{i}$ ). The range of the force given by the Compton wavelength of the moduli is independent of the number of large dimensions but is strongly dependent on the SUSY breaking scale and the string scale.

Of course, the presence of these moduli forces is not a model independent prediction. There may be string models with discrete torsion in which most or all of the twisted moduli are projected out. In addition, even if there are such twisted moduli in a given model they may obtain masses of $\sim M_{s}$ in which case such forces are completely negligible. However, it is interesting to see that there are generic scenarios with such forces with experimentally observable effects.

\section{Acknowledgements}

I would like to thank Jaume Gomis and Nemanja Kaloper for very useful discussions. 


\section{REFERENCES}

1. N. Arkani-Hamed, S. Dimopoulos and G. Dvali, Phys. Rev. Lett. B429 (1998) 263, hep-ph/9803315; I. Antoniadis, N. Arkani-Hamed, S. Dimopoulos and G. Dvali, Phys. Lett. B436, 257 (1998), hep-ph/9804398.

2. I. Antoniadis, Phys. Lett. B246 (1990) 377; J. Lykken, Phys. Rev. D54 (1996) 3693, hep-th/9603133; I. Antoniadis and K. Benakli, Phys. Lett. B326 (1994) 69; I. Antoniadis, K. Benakli and M. Quiros, Nucl. Phys. B331 (1994) 313.

3. N. Arkani-Hamed, S. Dimopoulos and G. Dvali, hep-ph/9807344.

4. S. Cullen and M. Perelstein, hep-ph/9903422.

5. L. Hall and D. Smith, hep-ph/9904267.

6. K.R. Dienes, E. Dudas and T. Gherghetta, Phys. Lett. B436, 55 (1998) hepph/9803466; R. Sundrum, hep-ph/9805471, hep-ph/9807348; G. Shiu and S.H. Tye, Phys. Rev. D58, 106007 (1998) hep-th/9805157; P.C. Argyres, S. Dimopoulos and J. March-Russell, Phys. Lett. B441, 96 (1998) hepth/9808138; N. Arkani-Hamed, S. Dimopoulos and J. March-Russell, hepth/9809124; A. Donini and S. Rigolin, hep-ph/9901443; Z. Berezhiani and G. Dvali, hep-ph/9811378; Z. Kakushadze, hep-th/9812163, hep-th/9902080; Z. Kakushadze and S.-H.H. Tye, hep-th/9809147; N. Arkani-Hamed, S. Dimopoulos, hep-ph/9811353; N. Arkani-Hamed, S. Dimopoulos, G. Dvali and J. March-Russell, hep-ph/9811448; P. Mathews, S. Raychaudhuri and K. Sridhar, hep-ph/9811501; hep-ph/9812486; A. Pomarol and M. Quiros, Phys. Lett. B438, 255 (1998); T.E. Clark and S.T. Love, hep-th/9901103;

T. Banks, M. Dine and A. Nelson, hep-th/9903019.

7. G.F. Giudice, R. Rattazzi and J.D. Wells, hep-ph/9811291; T. Han, J.D. Lykken and R. Zhang, hep-ph/9811350; J.L. Hewett, hep-ph/9811356; E.A. Mirabelli, M. Perlstein and M.E. Peskin, hep-ph/9811337; P. Mathews, S. 
Raychaudhuri and K. Srihar, hep-ph/9812486, hep-ph/9811501; I. Antoniadis and C. Bachas, hep-th/9812093; M.L. Graesser, hep-ph/9902310; S. Nussinov and R.E. Shrock, hep-ph/9811323; T.G. Rizzo, hep-ph/9901209; hep-ph/9902273; hep-ph/9903475; K. Agashe and N.G. Deshpande, hepph/9902263; K. Cheung, hep-ph/9904266; K. Cheung and W. Keung, hepph/9903294; M. Masip and A. Pomarol, hep-ph/9902467.

8. K. Benakli, hep-ph/9809582; K. Benakli and S. Davidson, hep-ph/9810280; M. Maggiore and A. Riotto, hep-th/9811089; D.H. Lyth, Phys. Lett. B448 (1999) 191, hep-ph/9810320; N. Kaloper and A. Linde, hep-th/9811141; G. Dvali and S.-H. H. Tye, hep-ph/9812483; K.R. Dienes, E. Dudas, T. Ghergetta, A. Riotto, hep-ph/9809406; N. Arkani-Hamed, S. Dimopoulos, N. Kaloper and J. March-Russell, hep-ph/9903239.

9. I. Antoniadis, S. Dimopoulos and G. Dvali, Nucl. Phys. B516 (1998) 70.

10. S. Dimopoulos and G. Giudice, hep-ph/9602350.

11. I. Antoniadis, hep-ph/9904272.

12. J. Long, H. Chan and J. Price, Nucl. Phys. B539 (1999) 23, and references therein.

13. G. Aldazabal, A. Font, L. Ibanez and G. Violero, hep-th/9804026.

14. E. Halyo, hep-ph/9901302.

15. G. Shiu, R. Schrock and S.-H. Tye, hep-ph/9904262.

16. L. Ibanez, C. Munoz and S. Rigolin, hep-ph/9812397.

17. L. Ibanez, R. Rabadan and A. Uranga, hep-th/9808139. 